\title{
From optochemical sensors for industrial processes to large-area printing of sensor systems integrated with organic electronics.
}

Köstler, Stefan ${ }^{1, *} ;$ Kraker, Elke $^{1}$; Sagmeister, Martin ${ }^{1}$; Lamprecht Bernhard ${ }^{1}$; Bizzarri, Alessandro ${ }^{1}$; Abel, Tobias $^{2}$; Mayr, Torsten ${ }^{2}$; Stadlober, Barbara ${ }^{1}$; Ribitsch, Volker ${ }^{1}$

1 JOANNEUM RESEARCH Forschungsgesellschaft $\mathrm{mbH}$

MATERIALS - Institute for Surface Technologies and Photonics

Steyrergasse 17, A-8010 Graz, Austria

stefan.koestler@joanneum.at

${ }^{2}$ Graz University of Technology

Institute of Analytical and Food Chemistry

Stremayrgasse 9/II, A-8010 Graz, Austria

\section{Principle setup of optical chemosensors:}

The importance of sensors to determine chemical, biochemical and metabolic parameters increases steadily in different fields of applications. Fast and reliable sensors are demanded e.g. for process control in industry, food- and biotechnology, patient monitoring, diagnostics, or environmental monitoring. Key performance parameters of such devices are sensitivity, selectivity and response time. Many optical chemosensors rely on the detection of luminescence signals which depend on the concentration of a certain chemical species (e.g. $\mathrm{O}_{2}, \mathrm{CO}_{2}, \mathrm{pH}, \ldots$ ). Luminescence detection is often preferred over other optical detection schemes (e.g. absorption) due to its superior sensitivity and selectivity [1].

Generally the concentration dependent luminescence properties of a sensor film in contact with the sample medium are detected by an optical system (Figure 1). Such optoelectronic readout systems consist of: a light source (e.g. light emitting diode, LED) for excitation of the dye, a photodetector for the detection of the emitted luminescence, some optical components like filters for separation of excitation and emission light, and a signal processing electronics [2]. A few examples of optoelectronic readout devices for oxygen sensors, designed for different applications in chemical process control and food packaging, are shown in Figure 2.

The sensitive films are basically composites of multiple polymer layers (see Figure 1b). For the carrier substrates usually transparent polymer films (e.g. PET) are chosen although in some applications glass can also be used. The luminescent sensing layer itself consists of a polymeric matrix material doped with a luminescent indicator dye. The sensitivity of the sensor as well as the selectivity for a certain analyte are mainly determined by the properties of these two materials. The chemical nature of the dye determines how its luminescence properties change upon interaction with the analyte (quenching, protonation/deprononation, electron transfer, ...). The polymeric matrix material is responsible for controlling the permeability for the analyte, interfering sample components and possible analyte enrichment. Depending on the desired analyte, measurement range and application field, the sensitive layer may also contain further components in order to modify the sensors selectivity, response characteristic, or other properties (e.g. phase transfer agents, plasticizers, ionophors).

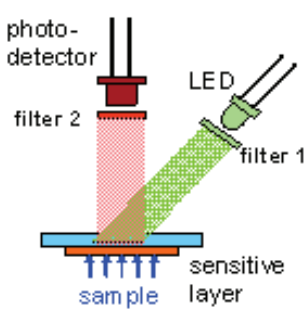

a)

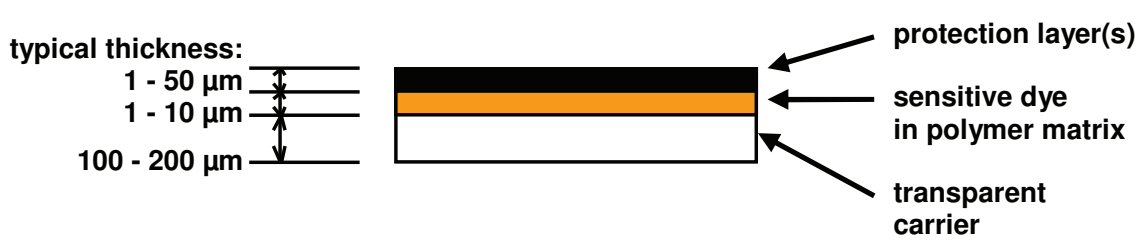

b)

Figure 1: a) Principle of optical chemosensors, b) Typical structure of luminescent sensor films.

While the sensitive layer alone would in principle be sufficient to provide the basic sensing functionality, in most cases one or more further layers are required to meet the requirements of specific applications. Such additional layers are responsible for chemical and/or mechanical protection, optical insulation, 
adhesion promotion, or to impart biocompatibility or anti-fouling properties to the sensor film. So in the design of optical sensor films the compatibility of all the different materials, processing techniques and adhesion issues have to be taken into account. Furthermore one has to bear in mind that the complete multilayer stack determines typical sensor performance parameters like e.g. response time.

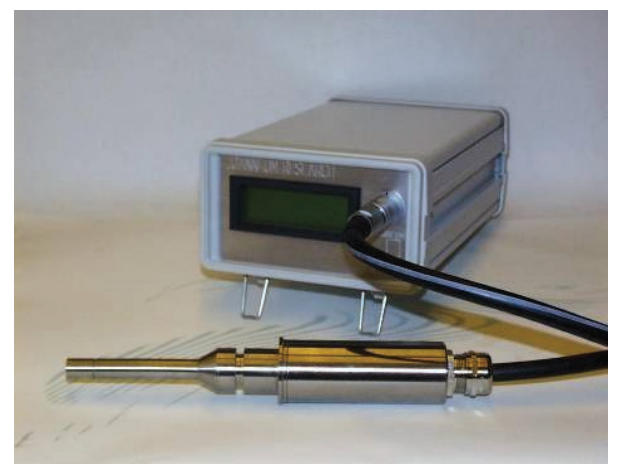

a)

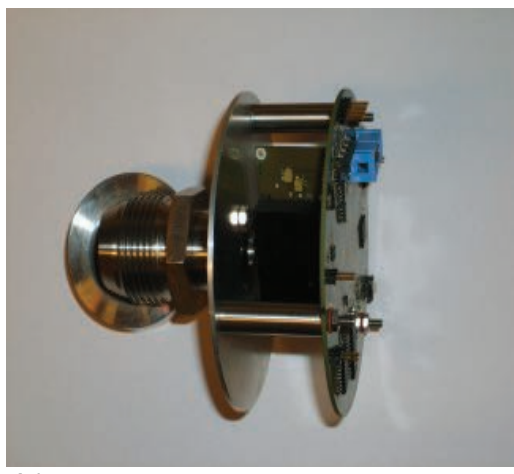

b)

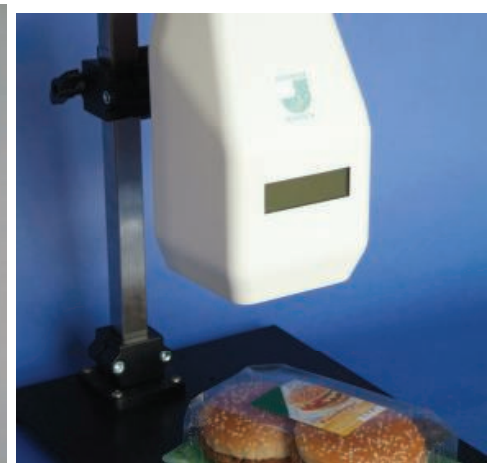

c)

Figure 2: a) Optical oxygen sensor probe and b) inline oxygen measurement system for industrial process control applications c) Instrument for non-destructive read-out of oxygen sensitive labels in food packages.

\section{Sensor film fabrication:}

The luminescent sensor films are either intimately attached to the readout system as replaceable components (for single or multiuse) or incorporated into a separate object directly at the measurement site (food packages, wound dressing, etc.). In the latter case the sensor spots are excited and read-out optically without direct contact to the optoelectronic unit. An example for the incorporation of sensor spots into packaging materials to monitor the atmosphere composition inside food packages is shown in Figure 3.

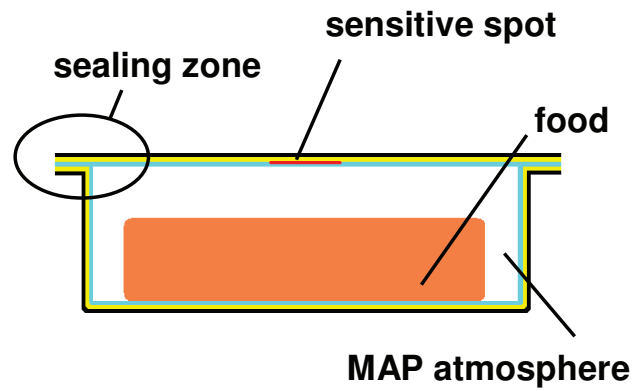

a)

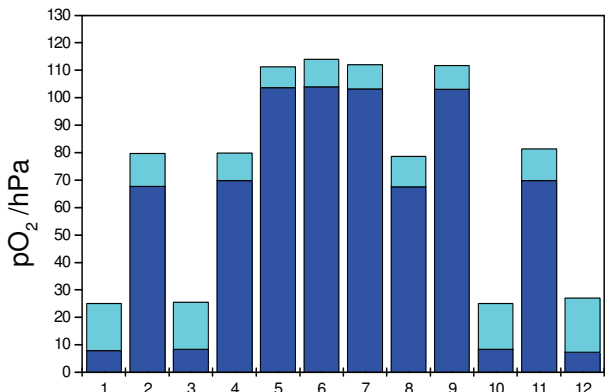

Package No.

Figure 3: a) Modified atmosphere packaged (MAP) food with incorporated sensor spot and b) oxygen levels one and seven days after MAP.

Such sensor systems allow non-destructive, continuous monitoring and give quantitative information on the atmosphere composition (e.g. $\mathrm{O}_{2}$ or $\mathrm{CO}_{2}$-levels) in retail food packs (meat, cheese, fish ...) and botteled beverages (beer, water, ...). This allows controlling of food packages as a whole directly after packaging and a random inspection along the distribution chain. Thus, such sensor systems can be used to detect problems related to the packaging process (sealing problems, poor gas barrier properties, pinholes, etc.), to monitor food freshness and detect spoilage during transport and distribution. Considering that nowadays modified atmosphere packaging (MAP) of food is applied on a scale of multiple billions of pieces per year [3] incorporation of sensor spots in such materials requires high volume fabrication technologies. Beside food packaging, other possible application areas requiring large numbers of optical sensors films are e.g. disposable diagnostic devices or smart textiles. For such high volume applications of optical sensors, additional requirements such as simple operation, high reproducibility and low-cost production are crucial factors. To meet these requirements, it is necessary to develop strategies for an intimate integration of components and the implementation of advanced and cost-efficient fabrication technologies. Fabrication technologies play a key role as performance and reproducibility of such sensor films strongly depend on fabrication related parameters like distribution of 
dyes in the film and between different polymer phases, thickness homogeneity, or adhesion between the different layers.

We have evaluated several approaches to sensor fabrication by the use of different printing processes for the deposition and patterning of functional sensor materials:

- Pad printing

- Screen printing

- Inkjet printing

- Offset printing

The tested printing techniques cover a wide range of possibilities with regard to achievable features size throughput, and flexibility. Inkjet printing for example allows relatively narrow structures below $100 \mu \mathrm{m}$ of thin films and almost unlimited flexibility in terms of fast pattern variation. Although inkjet printing can nowadays be integrated in roll-to-roll based production lines, it is still considered a relatively lowthroughput method compared to e.g. offset printing which is the preferred technique for graphic printing of packaging materials, newspapers or books. The different techniques also demand quite different printing ink formulations of the functional sensor materials with regard to viscosity, surface tension, solvent volatility, etc.. Table 1 shows for example the viscosity ranges suitable for the different printing techniques.

Table 1: Viscosity ranges for printing poly(styrene) based oxygen sensitive structures using different printing techniques:

\begin{tabular}{|l|l|l|l|l|}
\hline Printing technique & Pad printing & Screen printing & Inkjet printing & Offset printing \\
\hline Viscosity [Pa.s] & $0.075-0.25$ & $0.15-7.5$ & $0.005-0.010$ & $0.06-0.1$ \\
\hline
\end{tabular}

Especially for food packaging applications, sensor fabrication using standard offset printing is desirable because it is a standard technology for graphic printing on food packaging materials. Therefore, sensor fabrication using the same method facilitates integration into the production processes, lowers barriers of establishing the new technology and minimizes costs of sensor integration. Furthermore, offset printing is a continuous roll-to-roll fabrication technology that is one of the highest throughput techniques among printing methods and is suitable for large area patterning. Examples of oxygen sensor spots printed on a standard food packaging foil using an industrial offset machine are shown in Figure 4.

a)
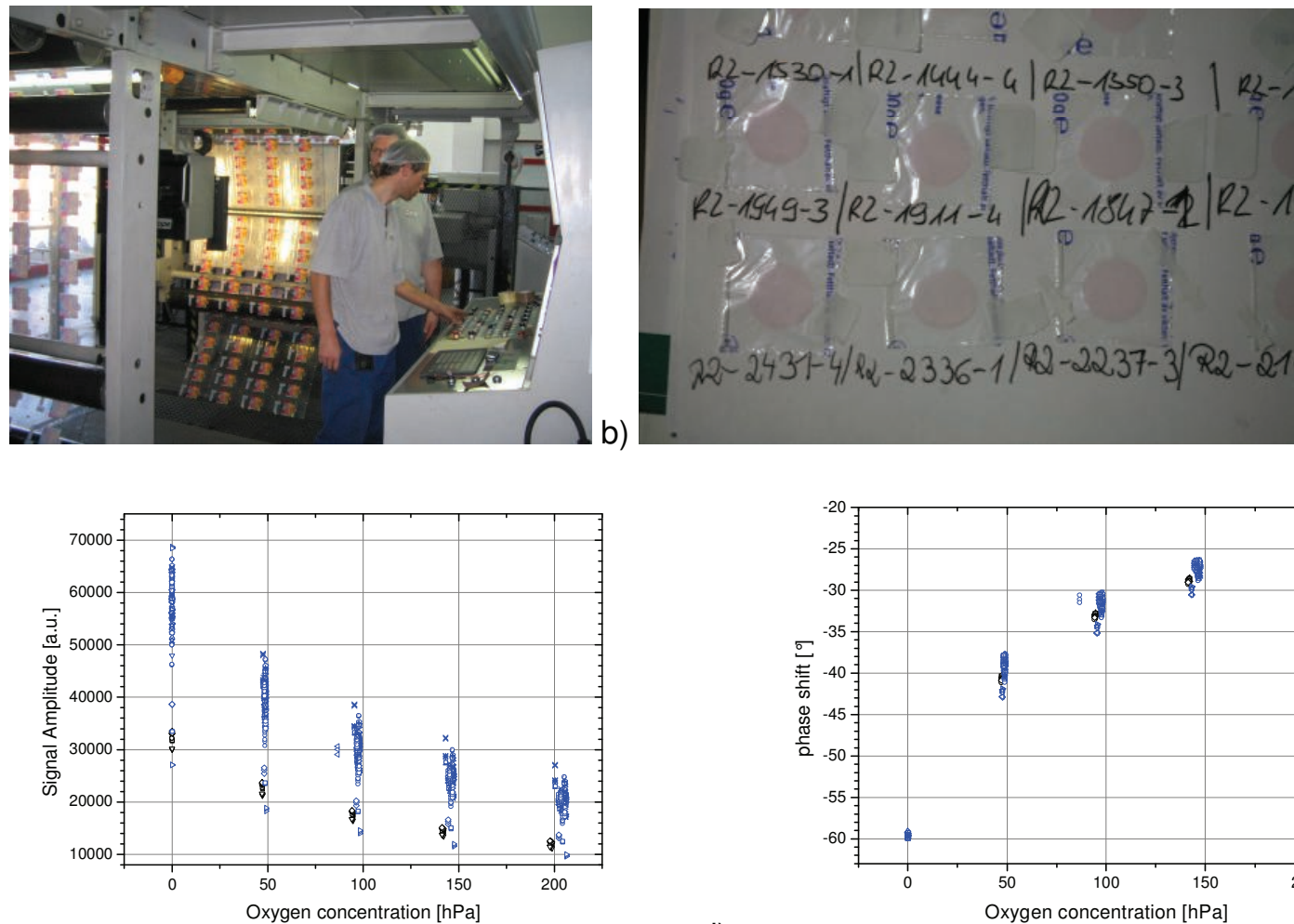

c)

d)

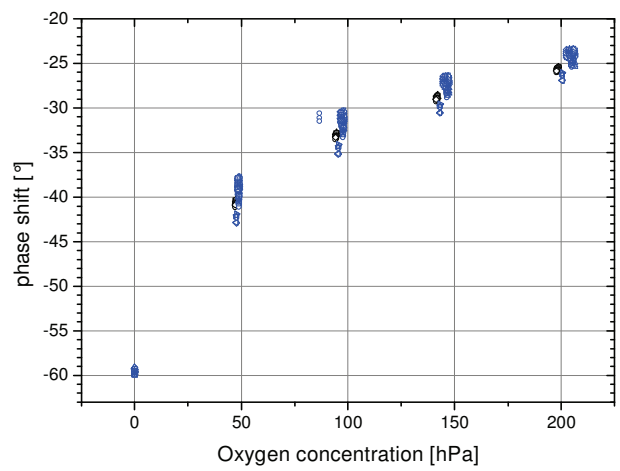

Figure 4: a) Offset printing of oxygen sensor spots on food packaging foil; b) Sensor spots cut-out for further characterization; c) luminescence intensity and d) luminescence decay-time proportional phase shift of oxygen sensor batches from different printing experiments. 
The detailed characterization of luminescence properties at different oxygen concentrations reveals that luminescence intensity values of the individual are quite scattered (Figure 4c). In contrast, phase shift values (which are a measure for luminescence decay time) show very similar behavior even for sensor spots prepared in different printing experiments (Figure 4d). One can conclude that for luminescence decay time based measurement, oxygen sensor spots can be printed in large volumes with very good reproducibility using industrial offset printing technology.

Nevertheless, for technology or materials development purposes, and for applications not requiring such large numbers of sensor spots, offset printing lacks flexibility. Here screen printing is a promising alternative. Screen printing enables the fast preparation of small batches of sensor samples from a broad variety of materials. Batch screen printing experiments can be performed with small amounts of inks and with minimal material loss what is important as inks for optical sensor fabrication usually contain pretty expensive materials (luminescent sensor dyes). Nevertheless, screen printing can be fully adapted for continuous roll-to-roll based manufacturing. Therefore, it has been the preferred technique in current attempts for the production of polymeric electronic devices like solar cells [4]. Figure 5 demonstrates some examples of screen printed optical sensors on polymer substrates, showing reproducible fabrication of high quality films. In order to realize novel sensor geometries using the polymeric patterns as active sensor waveguides [5] we could also demonstrate the fabrication of multilayer stacks containing integrated optical waveguides using screen printing technology [6].

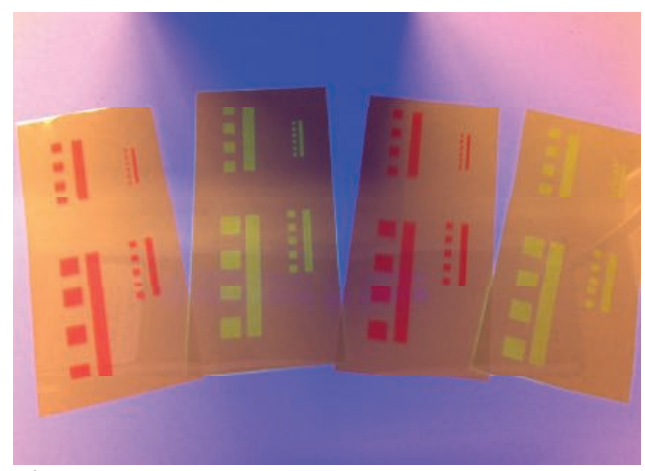

a)

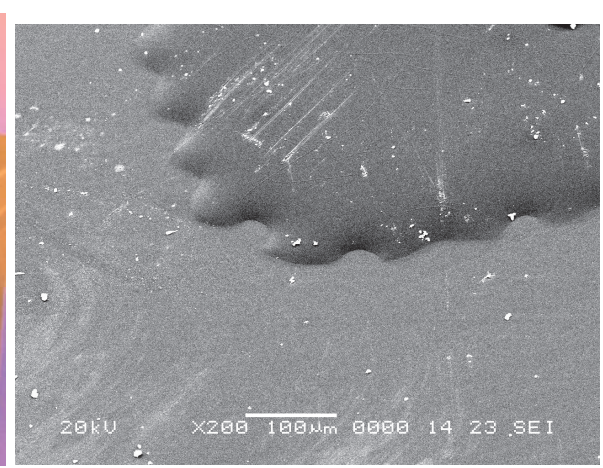

b)

Figure 5: a) Optical chemosensors prepared by screen printing. b) Electron microscopy image of screen printed sensor spot.

\section{Monolithic integration concepts:}

So far it has been shown that the luminescent polymer films of optical chemosensors can be fabricated by potentially low-cost, high volume printing technolgies. A next step towards mass producible, low-cost complete sensor systems is the integration of organic (opto)electronic devices with sensor functionalities. Such devices, based on organic semiconductor materials, can be built on a wide range of different substrates and principally lend themselves to solution based fabrication processes or layer-by-layer vacuum deposition. In the ideal case, this leads to the concept of monolithic integration of all components of a sensor system on a common substrate resulting in an autonomous device. In the case of optical chemosensors this requires the integration of a light source, a photodetector, the sensitive layers and optionally further components like microfluidics, electronic circuitry, optical components etc. to give a so called lab-on-a-chip or micro-total analysis system ( $\mu$-TAS).

We realized luminescence based chemosensor devices by integration of luminescent sensor elements with organic photodetectors (OPD) and organic light emitting diodes (oLEDs). The crucial task of efficient discrimination between the emitted luminescence and excitation light was accomplished using either polarization filters as substrates [7] or a special geometrical arrangement of components [8].

The latter consists of an array of ring shaped organic photodiodes fabricated on one side of a polymeric substrate. On the opposite side of the substrate an array of luminescent sensor spots is deposited in the center of the photodiodes by screen printing. For excitation of the sensor spots, a (organic) LED together with an aperture in order to avoid direct illumination of the detectors, is attached to the substrate (Figure 6). The substrate serves as a waveguide and guides the luminescence to the ring shaped photodiodes while excitation light cannot be coupled into the waveguide. An example of intensity and decay time based oxygen sensing with such a chip is shown in Figure 6c. Such sensor-chips are suitable for multianalyte detection of parameters like $\mathrm{O}_{2}, \mathrm{CO}_{2}, \mathrm{pH}, \mathrm{NH}_{3}$, etc. if the respective sensitive spots are 
printed on the photodiode array. They can furhter be integrated into microfluidic structures and thus makes them interesting for bioanalytical applications.

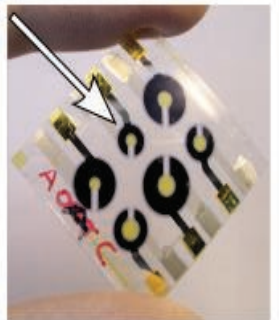

a)

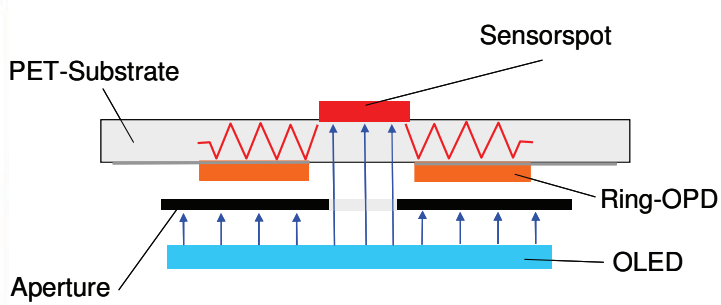

b)

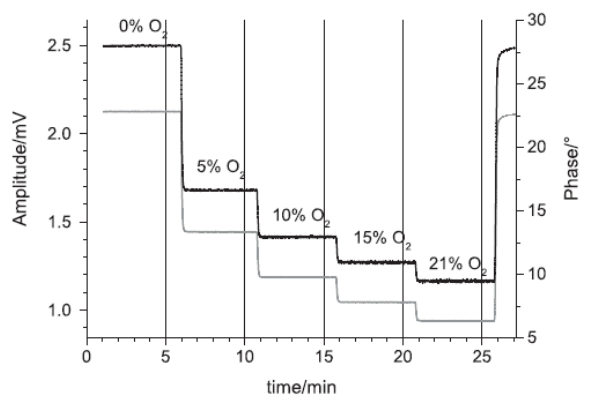

c)

Figure 6: a) Sensor chip comprising six optical sensor dye spots integrated with ring shaped organic photodiodes (OPDs) b) Schematic setup of optical chemosensor realized with integrated organic optoelectronic devices c) Response curve of integrated oxygen sensor chip [8] (๑ WILEY-VCH Verlag GmbH \& Co. KGaA, Weinheim).

In another example, the monolithic integration of sensor devices with organic transistors could be shown. In this set-up, a physical sensor with an integrated pyroelectric polymer sensing element and an organic thin film transistor on a common substrate was designed (Figure 7) [9]. The pyroelectric capacitor element is used to switch the transistor via a common gate electrode and is highly sensitive to changes in ambient temperature. Therefore this device can be used for infrared detection and to detect human body movements (e.g. in human-machine interfaces). Recently, a system consisting of an array of capacitive sensing units, organic transistors for signal processing and an organic display unit could be realized in which all components were fabricated by screen printing on flexible substrates [10].

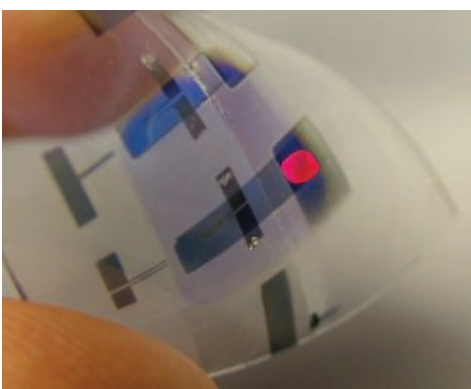

Figure 7: Polymer pyroelectric sensor units integrated with organic thin film transistors [9]. (@ WILEY-VCH Verlag GmbH \& Co. KGaA, Weinheim)

This successful fabrication of organic electronic based circuitry for sensor signal processing is a very promising result for the future development of monolithically integrated chemical sensor systems. It suggests, that in principle not only organic light sources and photodetectors but also some basic signal processing circuitry based on organic devices could be integrated into such sensor or lab-on-a-chipsystems.

Another important step towards the low-cost fabrication of monolithically integrated lab-on-chip systems can be expected from the fast progress in roll-to-roll processing technology. Previously applied methods (such as offset printing) were basically limited to relatively large patterns and the simple deposition of coatings. Recent developments of roll-to-roll equipment now allow continuous roll-to-roll based fabrication of micro- or even nanostructures by imprinting techniques on flexible polymer substrates (Figure 8). In combination with printing of functional materials this opens new opportunities for the integration of e.g. microfluidic structures, (opto)electronic devices, and sensors. Especially in the field of microfluidics, several groups have already successfully used roll-to-roll imprinting tools for the fabrication of polymer microfluidic devices [11, 12]. Thus, for the future one could imagine the fabrication of complete physical, chemical, and biochemical sensor systems in roll-to-roll based production lines. 


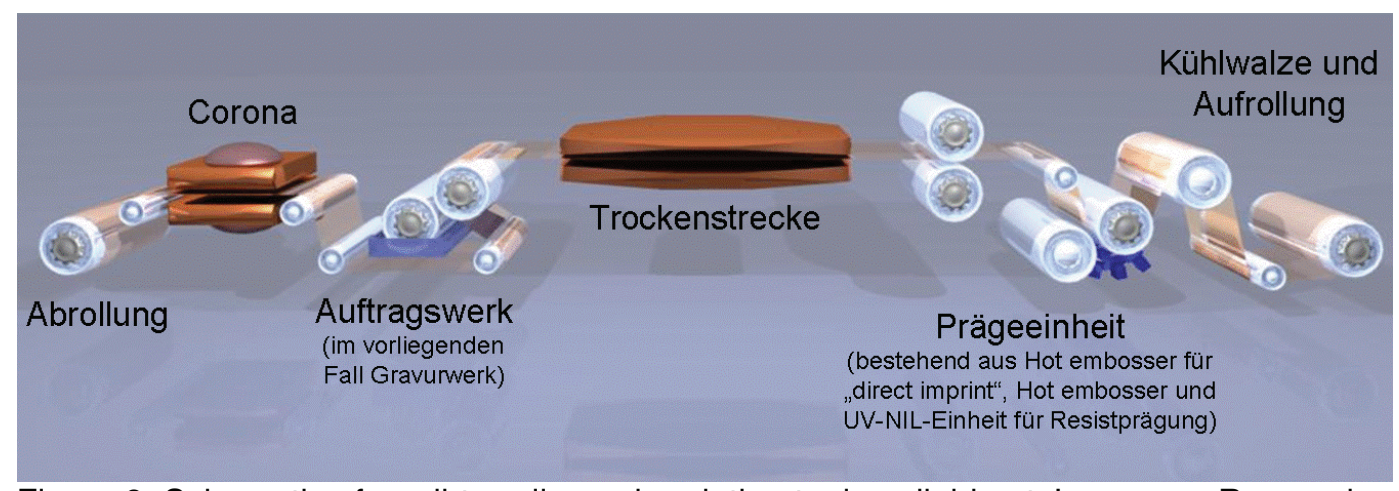

Figure 8: Schematic of a roll-to-roll nanoimprinting tool available at Joanneum Research.

Acknowledgements:

Financial support by the Austrian Research Promotion Agency (FFG) via the project cluster ISOTEC (Integrated organic sensor and optoelectronics technologies) and the Austrian Ministry for Transport, Innovation and Technology (BMVIT) via the Förderungsvereinbarung is gratefully acknowledged.

\section{References:}

1. McDonagh, C., C.S. Burke, and B.D. MacCraith, Optical Chemical Sensors. Chemical Reviews, 2008. 108(2): p. 400-422.

2. Cajlakovic, M., et al., Optochemical Sensors Based on Luminescence, in Encyclopedia of Sensors, E.C.D. Craig A. Grimes, and Michael V. Pishko, USA, Editor. 2006, American Scientific Publisher: 25650 North Lewis Way, Stevenson Ranch, California 91381-1439, USA. p. 291-314.

3. Mills, A., Oxygen indicators and intelligent inks for packaging food. Chemical Society Reviews, 2005. 34: p. 1003-1011.

4. Krebs, F.C., Fabrication and processing of polymer solar cells: A review of printing and coating techniques. Solar Energy Materials \& Solar Cells, 2009. 93: p. 394-412.

5. Mayr, T., et al., A planar waveguide optical sensor employing simple light coupling. Analyst, 2009. 134(8): p. 1544-1547.

6. Sagmeister, M., Screen printed polymer optical wavegiudes for integrated optics. 2011: p. submitted for publication.

7. Kraker, E., et al., Integrated organic electronic based optochemical sensors using polarization filters. Applied Physics Letters, 2008. 92(3): p. 033302.

8. Lamprecht, B., et al., Integrated fluorescence sensor based on ring-shaped organic photodiodes. physica status solidi - Rapid Research Letters, 2010. 4(7): p. 157-159.

9. Zirkl, M., et al., Low-Voltage Organic Thin-Film Transistors with high-k Nanocomposite Gate Dielectrics for Flexible Electronics and Optothermal Sensors. Advanced Materials, 2007. 19: p. 2241-2245.

10. Zirkl, M., et al., All-printed active matrix ferroelectric sensor network enabling touchless control and based on only five functional materials. Advanced Materials, 2011. 23: p. accpted.

11. Laurberg Vig, A., et al., Roll-to-roll fabricated lab-on-a-chip devices. Journal of Micromechanics and Microengineering, 2011. 21: p. 035006.

12. Velten, T., et al., Microfluidics on foil: state of the art and new developments. Proceedings of the Institution of Mechanical Engineers. Part B, Journal of Engineering Manufacture, 2008. 222(1): p. 107-116. 